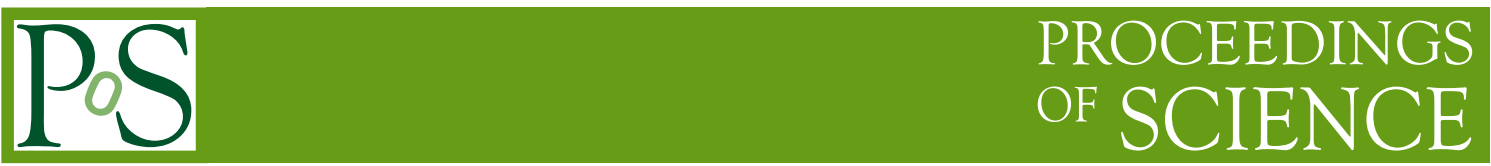

\title{
In medium Langevin dynamics of heavy quarkonium
}

\author{
Peter Vander Griend ${ }^{a * *}$ \\ ${ }^{a}$ Physik-Department, Technische Universität München, James-Franck-Str. 1, 85748 Garching, Germany \\ E-mail: vandergriend@tum.de
}

Heavy quarks and their bound states are ideal probes of the quark gluon plasma formed in relativistic heavy ion collisions. Due to the hierarchy of scales of the system, the in-medium dynamics of heavy quarkonium can be modeled by a Langevin equation in which uncorrelated interactions with the medium alter the momentum of the bound state. The hierarchy of scales makes the problem ideally suited for the use of effective field theories, namely potential nonrelativistic QCD, and the formalism of open quantum systems. We utilize these tools to perform a first principles treatment of heavy quarkonium in medium and analyze the regimes in which the dynamics take the form of a Langevin equation.

\footnotetext{
*** 10th International Workshop on Charm Physics (CHARM2020), ***

*** 31 May - 4 June, 2021 ***

*** Mexico City, Mexico - Online ***
}

\footnotetext{
${ }^{*}$ Speaker
} 


\section{Introduction}

Heavy ion collisions and the hot, dense medium they create provide unprecedented access to physical systems with dynamics resembling those of the very early universe in the first instants after the big bang. This hot, dense medium may in fact be quark gluon plasma (QGP) in which the color charged degrees of freedom, namely quarks and gluons which at low energies and temperatures are confined inside hadrons, exist freely. As such, identification of theoretically calculable and experimentally measurable observables is a task of great importance. Due to their heavy mass relative to the energy scales of the plasma, heavy quarks are theorized to provide such observables. Specifically, the hierarchy of scales $M \gg \pi T$, where $M$ is the heavy quark mass and $T$ is the temperature of the plasma, makes a Langevin equation a natural candidate to describe the dynamics of an in-medium bound state consisting of a heavy quark and a heavy antiquark. In this conference proceeding, we present the first principles derivation of a Langevin equation describing the in-medium dynamics of a heavy-heavy bound state using the effective field theory potential nonrelativistic QCD (pNRQCD) and the formalism of open quantum systems (OQS). The remainder of this proceedings is organized as follows: in section 2, we provide theoretical background to the problem including the relevant hierarchy of scales, effective field theory methods, the formalism of open quantum systems, and Langevin dynamics; in section 3, we present the evolution equations of in-medium heavy quarkonium and derive a Langevin equation; we conclude in section 4.

\section{Theoretical background}

In this section, we present the theoretical background necessary to describe the in-medium dynamics of heavy quarkonium and derive a Langevin equation. In subsection 2.1, we discuss the hierarchy of scales of the problem; in subsections 2.2 and 2.3, we present the effective field theories and open quantum system methods used to describe the system, respectively; and in subsection 2.4, we present Langevin dynamics.

\subsection{Scales of the problem}

We consider heavy charmonium, i.e., the bound state of a charm and an anti-charm quark, propagating in the QGP formed in a heavy ion collision. A bound state consisting of a heavy quark and a heavy antiquark is characterized by (at least) three energy scales: the heavy quark mass $M$, the inverse of the Bohr radius $1 / a_{0}$, and the binding energy $E$. We take the pole mass of the charm quark $M_{c}=1.67 \mathrm{GeV}$ [1]. The Bohr radius $a_{0}$ is calculated by solving

$$
a_{0}=\frac{2}{C_{F} \alpha_{s}\left(1 / a_{0}\right) M_{c}}
$$

where $C_{F}$ is the quadratic Casimir of the fundamental representation $C_{F}=\left(N_{c}^{2}-1\right) /\left(2 N_{c}\right)$ for $N_{c}=3$ colors and $\alpha_{s}\left(1 / a_{0}\right)$ is the strong coupling evaluated at the inverse of the Bohr radius with the 1-loop running and $\Lambda_{\frac{M S}{n_{f}}=3}=332 \mathrm{MeV}[2,3]$ where $n_{f}=3$ is the number of fermions. This gives an inverse Bohr radius of $1 / a_{0}=0.839 \mathrm{GeV}$ and a Coulombic binding energy of $E=-0.421$ $\mathrm{GeV}$. The heavy quarkonium is Coulombic in medium if $1 / a_{0} \gg(\pi T)$; the Coulombic description is thus valid for charmonium in GQP at temperatures $T \lesssim 275 \mathrm{MeV}$. 


\subsection{Effective field theory methods}

Effective field theory methods allow for a systematic exploitation of inherent hierarchies of scale present in a physical system. pNRQCD is an effective field theory of the strong interaction describing the dynamics of a heavy-heavy QCD bound state. It takes advantage of the large heavy quark mass $M$, the resulting nonrelativistic nature of the system, i.e., $v \ll 1$, where $v$ is the relative velocity, and the small radius of the bound state. Integrating out the hard scale $M$ from full QCD, one obtains nonrelativistic QCD (NRQCD) [4,5]; further integrating out the soft scale $M v$ one obtains pNRQCD [6-8]. The degrees of freedom of the resulting theory are are composite fields representing the heavy-heavy bound state in the singlet and octet color configurations and gluons and light quarks at the ultrasoft scale $M v^{2}$; interactions take the form of chromoelectric dipole vertices mediating singlet-octet and octet-octet transitions. The information of the soft gluons is encoded in an attractive singlet and repulsive octet potential

$$
V_{s}=-\frac{N_{c}^{2}-1}{2 N_{c}} \frac{\alpha_{s}}{r}, \quad V_{o}=\frac{1}{2 N_{c}} \frac{\alpha_{s}}{r},
$$

where $N_{c}$ is the number of colors, $\alpha_{s}$ is the strong coupling, and $r$ is the bound state radius. At leading order, the equations of motion of the singlet and octet fields are given by Schrödinger equations in which the singlet and octet Hamiltonians consist of the sum of a kinetic term and the corresponding potential

$$
h_{s, o}=\frac{\mathbf{p}^{2}}{M}+V_{s, o} .
$$

The resulting Lagrangian implements an expansion in the bound state radius $r$ and the inverse of the heavy quark mass $M^{-1}$.

\subsection{Open quantum systems}

The formalism of open quantum systems allows for the rigorous treatment of a quantum system of interest (here the heavy quarkonium) coupled to an environment (here the QGP); for a general introduction to OQS methods, see Ref. [9]. The total system consisting of the system and the environment is characterized by three time scales: the system time scale $\tau_{S}$, the environment time scale $\tau_{E}$, and the relaxation time $\tau_{R}$. For in-medium heavy quarkonium with binding energy $E$ and Bohr radius $a_{0}$ in a plasma at temperature $T$,

$$
\tau_{E} \sim E^{-1}, \quad \tau_{R} \sim(\pi T)^{-1}, \quad \tau_{R} \sim \Sigma_{s}^{-1} \sim\left(a_{0}^{2}(\pi T)^{3}\right)^{-1},
$$

where $\Sigma_{S}$ is the heavy quarkonium thermal self energy. The combined system under consideration realizes $\tau_{R}, \tau_{S} \gg \tau_{E}$ qualifying the evolution as quantum Brownian motion and justifying the use of the Born and Markov approximations. The Born approximation consists in taking the quarkonium to have little effect on the plasma at time scales of interest. This implies neither a weak coupling between the quarkonium and the plasma nor that the quarkonium causes no excitations in the plasma, but rather, a course graining to time scales over which any excitations in the plasma have relaxed back. At the calculational level, the Born approximation implies the factorization of the total density matrix $\rho(t)$ into the tensor product of the density matrix of the system $\rho_{S}(t)$ and the density matrix of the environment $\rho_{E}$ (and taking the environment density matrix as constant 
in time), i.e., $\rho(t) \propto \rho_{S}(t) \otimes \rho_{E}$. The Markov approximation implies the temporal locality of the evolution equations of the system, i.e., that the evolution of the quarkonium at time $t$ depends only on the state of the quarkonium at time $t$, thus eliminating a memory integral from the evolution equations.

\subsection{Langevin dynamics}

The dynamics of a particle interacting with a medium via so-called "kicks" which alter the particle's momentum are described by Langevin equations

$$
\frac{\mathrm{d} p_{i}}{\mathrm{~d} t}=-\eta_{D} p_{i}+\xi_{i}(t), \quad\left\langle\xi_{i}(t) \xi_{j}\left(t^{\prime}\right)\right\rangle=\kappa \delta_{i j} \delta\left(t-t^{\prime}\right),
$$

where $p_{i}$ is the three momentum of the particle, $\eta_{D}$ is the drag coefficient, $\xi_{i}$ represents the random force on the particle due to the "kicks" from the medium, and $\kappa$ is the momentum diffusion coefficient. The drag and momentum diffusion coefficients are related by the Einstein relation

$$
\eta_{D}=\frac{\kappa}{2 M T} .
$$

Langevin dynamics is a natural candidate to describe the in-medium dynamics of a heavy quark of mass $M$ in a plasma at temperature $T$ where $M \gg \pi T$. In Ref. [10], the form of $\kappa$ for a single heavy quark in medium was derived

$$
\kappa=\frac{g^{2}}{6 N_{c}} \int_{0}^{\infty} \mathrm{d} s\left\langle\left\{\tilde{E}^{a, j}(s, \mathbf{0}), \tilde{E}^{a, j}(0, \mathbf{0})\right\}\right\rangle,
$$

where

$$
\tilde{E}^{a, j}(s, \mathbf{0})=\Omega(s) E^{a, i}(s, \mathbf{0}) \Omega^{\dagger}(s), \quad \Omega(s)=\exp \left[-i g \int_{-\infty}^{s} \mathrm{~d} s^{\prime} A_{0}\left(s^{\prime}, \mathbf{0}\right)\right] .
$$

\section{Evolution Equations}

In this section, we present the evolution equations of in-medium, heavy quarkonium and derive a Langevin equation.

\subsection{Master and Lindblad equations}

The in-medium, nonequilibrium evolution equations of heavy quarkonium in a strongly coupled medium were derived in Refs. [11, 12] and take the form of a master equation

$$
\frac{\mathrm{d} \rho(t)}{\mathrm{d} t}=-i[H, \rho(t)]+\sum_{n, m} h_{n m}\left(L_{i}^{n} \rho(t) L_{i}^{m \dagger}-\frac{1}{2}\left\{L_{i}^{m \dagger} L_{i}^{n}, \rho(t)\right\}\right),
$$

where

$$
\begin{aligned}
& \rho(t)=\left(\begin{array}{cc}
\rho_{s}(t) & 0 \\
0 & \rho_{o}(t)
\end{array}\right), \quad H=\left(\begin{array}{cc}
h_{s}+\operatorname{Im}\left[\Sigma_{s}\right] & 0 \\
0 & h_{o}+\operatorname{Im}\left[\Sigma_{o}\right]
\end{array}\right), \quad L_{i}^{0}=\left(\begin{array}{ll}
0 & 0 \\
0 & 1
\end{array}\right) r^{i}, \\
& L_{i}^{1}=\left(\begin{array}{cc}
0 & 0 \\
0 & \frac{N_{c}^{2}-4}{2\left(N_{c}^{2}-1\right)} A_{i}^{o o^{\dagger}}
\end{array}\right), \quad L_{i}^{2}=\left(\begin{array}{cc}
0 & \frac{1}{\sqrt{N_{c}^{2}-1}} \\
1 & 0
\end{array}\right) r^{i}, \quad L_{i}^{3}=\left(\begin{array}{cc}
0 & \frac{1}{\sqrt{N_{c}^{2}-1}} A_{i}^{o s \dagger} \\
A_{i}^{s o \dagger} & 0
\end{array}\right) r^{i},
\end{aligned}
$$




$$
h_{n m}=\left(\begin{array}{llll}
0 & 1 & 0 & 0 \\
1 & 0 & 0 & 0 \\
0 & 0 & 0 & 1 \\
0 & 0 & 1 & 0
\end{array}\right), \quad A_{i}^{u v}=\frac{g^{2}}{6 N_{c}} \int_{0}^{\infty} \mathrm{d} s e^{-i h_{u} s} r^{i} e^{i h_{v} s}\left\langle\tilde{E}^{a, j}(0, \mathbf{0}) \tilde{E}^{a, j}(s, \mathbf{0})\right\rangle,
$$

where $u$ and $v$ take the values $s$ (for singlet) or $o$ (for octet) and

$$
\Sigma_{s}=r^{i} A_{i}^{s o \dagger}, \quad \Sigma_{o}=\frac{1}{N_{c}^{2}-1} r^{i} A_{i}^{o s \dagger}+\frac{N_{c}^{2}-4}{2\left(N_{c}^{2}-1\right)} r^{i} A_{i}^{o o \dagger} .
$$

The density matrix $\rho_{s, o}(t)$ represents the quarkonium in the color singlet, octet state. Interactions with the medium are fully encoded in the operator $A_{i}^{u v}$ of Eq. (12); the Wilson lines flanking the electric fields ensure the gauge invariance of $A_{i}^{u v}$ and of the master equation as a whole. We emphasize that Eq. (9) is explicitly quantum, nonabelian, and heavy quark number conserving and accounts for both dissociation and recombination.

The electric-electric correlator in $A_{i}^{u v}$ decays for times $s \gtrsim T^{-1}$ giving the integral support in the region $s \lesssim T^{-1}$. As the Hamiltonian $h_{s, o}$ is of the order of the binding energy, i.e., $h_{s, o} \sim E$, an expansion of the exponentials in $A_{i}^{u v}$ amounts to an expansion in $E / T$. Therefore, in the limit $(\pi) T \gg E$, the exponentials in $A_{i}^{u v}$ may be set to 1, i.e., $e^{-i h_{s, o} s} \approx 1$, and $A_{i}^{u v}$ reduces to a linear combination of the transport coefficients $\kappa$ and $\gamma$

$$
A_{i}^{u v}=\frac{r^{i}}{2}(\kappa-i \gamma)
$$

where

$$
\begin{aligned}
& \kappa=\frac{g^{2}}{6 N_{c}} \int_{0}^{\infty} \mathrm{d} s\left\langle\left\{\tilde{E}^{a, j}(s, \mathbf{0}), \tilde{E}^{a, j}(0, \mathbf{0})\right\}\right\rangle, \\
& \gamma=-i \frac{g^{2}}{6 N_{c}} \int_{0}^{\infty} \mathrm{d} s\left\langle\left[\tilde{E}^{a, j}(s, \mathbf{0}), \tilde{E}^{a, j}(0, \mathbf{0})\right]\right\rangle .
\end{aligned}
$$

As mentioned in subsection $2.4, \kappa$ is the heavy quark momentum diffusion coefficient occurring in a Langevin equation describing the in-medium diffusion of a single heavy quark; $\gamma$, first identified in Refs. [11, 12], is the dispersive counterpart of $\kappa$. In this limit, the master equation can be written in Lindblad form; previous works solved this Lindblad equation and extracted experimental observables including the nuclear modification factor $R_{A A}$ and the elliptic flow $v_{2}$ [13-15].

\subsection{Langevin equation}

In this subsection, we move beyond the strict $E \gg(\pi) T$ limit and show how these higher order corrections lead to a Langevin equation. Our procedure follows closely that of Blaizot and Escobedo who performed a similar calculation in Ref. [16] in an NRQCD rather than PNRQCD framework.

Moving to linear order in $E / T$, we write $A_{i}^{u v}$ as

$$
\begin{aligned}
A_{i}^{u v} & =\frac{g^{2}}{6 N_{c}} \int_{0}^{\infty} \mathrm{d} s\left(r^{i}-i s\left(h_{u} r_{i}-r_{i} h_{v}\right)+\cdots\right)\left\langle\tilde{E}^{a, j}(0, \mathbf{0}) \tilde{E}^{a, j}(s, \mathbf{0})\right\rangle \\
& =\frac{r_{i}}{2}(\kappa-i \gamma)+\kappa\left(-\frac{i p_{i}}{2 M T}+\frac{\Delta V_{u v}}{4 T} r_{i}\right)
\end{aligned}
$$


where $\Delta V_{u v}=V_{u}-V_{v}$ is the difference between the $u$ and $v$ (singlet $s$ and octet $o$ ) potentials, and we make use of the relation

$$
i \frac{g^{2}}{6 N_{c}} \int_{0}^{\infty} \mathrm{d} s s\left\langle\tilde{E}^{a, i}(s, \mathbf{0}) \tilde{E}^{a, i}(0, \mathbf{0})\right\rangle=\frac{\kappa}{4 T} .
$$

Following the procedure of Blaizot and Escobedo of Ref. [16], we project the evolution equations onto eigenstates of the radius of the heavy quarkonium $\langle\mathbf{r}|$ and $\left|\mathbf{r}^{\prime}\right\rangle ; \mathbf{r}$ and $\mathbf{r}^{\prime}$ can be considered to be the radius of the heavy quarkonium before and after, respectively, interaction with the medium. It is here convenient to switch to the coordinate system

$$
\mathbf{r}_{+}=\frac{\mathbf{r}+\mathbf{r}^{\prime}}{2}, \quad \mathbf{r}_{-}=\mathbf{r}-\mathbf{r}^{\prime}
$$

To extract the leading order evolution, we assign a scaling to the operators appearing in the evolution equations. The bound state is Coulombic implying the radius $\mathbf{r} \sim 1 / \sqrt{E M}$; we take this to be fulfilled before and after interaction with the medium implying

$$
\mathbf{r}_{+} \sim 1 / \sqrt{E M}, \quad \nabla_{+} \sim \sqrt{E M} .
$$

The potentials and their difference scale as the binding energy $E$

$$
V_{s, o}, \Delta V_{s o} \sim E \text {. }
$$

$\kappa$ and $\gamma$ are thermal quantities of dimension 3, so we take them to scale as the thermal scale cubed

$$
\kappa, \gamma \sim(\pi T)^{3} \text {. }
$$

Consistent with the Langevin picture in which the interaction with the medium thermalizes the heavy particle, we take the momentum associated with the change in radius to scale (at most) as the square root of the product of the thermal scale and the mass implying the following scaling

$$
\mathbf{r}_{-} \gtrsim 1 / \sqrt{\pi T M}, \quad \nabla_{-} \lesssim \sqrt{\pi T M} .
$$

We have three dimensionful parameters setting the scales of problem: $M,(\pi) T$, and $E$ fulfilling the hierarchy of scales $M \gg \pi T \gg E$. This allows for two small dimensionless parameters in which to expand: $E /(\pi T)$ and $(\pi T) / M$. In the following analysis, we take $E /(\pi T)$ and $(\pi T) / M$ to scale similarly, i.e., $E /(\pi T) \sim(\pi T) / M$. For bottomonium, this is fulfilled to a good degree of accuracy at very early times in central $\mathrm{Pb}-\mathrm{Pb}$ collisions at center of mass energy $\sqrt{s_{N N}}=2.76 \mathrm{TeV}$ and for charmonium at later times or in more peripheral collisions. As the plasma expands and cools, $E /(\pi T)$ begins to dominate over $(\pi T) / M$; nevertheless, in the following analysis, we assume $E /(\pi T)$ to scale similarly to $(\pi T) / M$. An analysis of the regime $E /(\pi T)>(\pi T) / M$ is beyond the scope of the current work and will be treated elsewhere [17]. For convenience, we define the expansion parameter

$$
\epsilon \sim \sqrt{E /(\pi T)} \sim \sqrt{(\pi T) / M}
$$

The leading order terms of the evolution equation are of order $\pi T$ and are given by

$$
\frac{\mathrm{d}}{\mathrm{d} t}\left(\begin{array}{l}
\rho_{s}^{\mathbf{r r}^{\prime}} \\
\rho_{o}^{\mathbf{r r}^{\prime}}
\end{array}\right)=\left(\begin{array}{cc}
-r_{+}^{2} \kappa & \frac{1}{N_{c}^{2}-1} r_{+}^{2} \kappa \\
r_{+}^{2} \kappa & -\frac{1}{N_{c}^{2}-1} r_{+}^{2} \kappa
\end{array}\right)\left(\begin{array}{c}
\rho_{s}^{\mathbf{r r}^{\prime}} \\
\rho_{o}^{\mathbf{r r}^{\prime}}
\end{array}\right)+\cdots,
$$


where $\rho_{s, o}^{\mathbf{r r}^{\prime}}=\left\langle\mathbf{r}\left|\rho_{s, o}(t)\right| \mathbf{r}^{\prime}\right\rangle$ and the ellipsis indicates terms suppressed by addition powers of $\epsilon$. The eigenvalues of the evolution matrix are

$$
\left\{\lambda_{0}, \lambda_{8}\right\}=\left\{0,-r_{+}^{2} \frac{N_{c}^{2}}{N_{c}^{2}-1}\right\} .
$$

The eigenvalue $\lambda_{0}$ corresponds to the maximum entropy state to which the system evolves at late times; the negative eigenvalue $\lambda_{8}$ corresponds to a state which decays to the maximum entropy state. Further following Blaizot and Escobedo, we diagonalize the leading order evolution equations by moving to the basis $\left\{\rho_{0}, \rho_{8}\right\}$ where

$$
\rho_{0}=\frac{\rho_{s}+\rho_{o}}{N_{c}^{2}}, \quad \rho_{8}=\frac{\left(N_{c}^{2}-1\right) \rho_{s}-\rho_{o}}{N_{c}^{2}},
$$

and include terms further suppressed in $\epsilon$

$$
\frac{\mathrm{d}}{\mathrm{d} t}\left(\begin{array}{c}
\rho_{0}^{\mathbf{r} \mathbf{r}^{\prime}} \\
\rho_{8}^{\mathbf{r} \mathbf{r}^{\prime}}
\end{array}\right)=\left(\begin{array}{cc}
\ell_{00}^{(1)}+\ell_{00}^{(2)} & \ell_{08}^{(1)}+\ell_{08}^{(2)} \\
\ell_{80}^{(1)}+\ell_{80}^{(2)} & \ell_{88}^{(0)}+\ell_{88}^{(1)}+\ell_{88}^{(2)}
\end{array}\right)\left(\begin{array}{c}
\rho_{0}^{\mathbf{r} \mathbf{r}^{\prime}} \\
\rho_{8}^{\mathbf{r r}}
\end{array}\right)+\cdots,
$$

where the subscripts indicate the quadrant of the evolution matrix, the superscripts in parenthesis indicate the further degree of suppression in $\epsilon$ with respect to the leading order evolution, and the ellipsis indicates further suppressed terms. We denote the eigenvalues of this evolution matrix $\lambda_{0}^{\prime}$ and $\lambda_{8}^{\prime}$ and note that in the limit $(\pi T) / M, E /(\pi T) \rightarrow 0$ they reduce to $\lambda_{0}$ and $\lambda_{8}$, respectively. We expand the eigenvalue $\lambda_{0}^{\prime}$ in $\epsilon$ finding

$$
\lambda_{0}^{\prime}=\ell_{00}^{(1)}+\ell_{00}^{(2)}-\frac{\ell_{08}^{(1)} \ell_{80}^{(1)}}{\ell_{88}^{(0)}}+\cdots,
$$

where the ellipsis indicates further suppressed terms and

$$
\begin{gathered}
\ell_{00}^{(1)}=\frac{2 i}{M} \nabla_{+} \cdot \nabla_{-}-\frac{i}{2} \mathbf{r}_{+} \cdot \mathbf{r}_{-} \gamma, \quad \ell_{00}^{(2)}=-\kappa\left(\frac{\mathbf{r}_{-} \cdot \nabla_{-}}{2 M T}+\frac{\mathbf{r}_{-}^{2}}{4}\right), \\
\ell_{08}^{(1)}=-\frac{1}{N_{c}^{2}-1} \frac{i}{2} \mathbf{r}_{+} \cdot \mathbf{r}_{-} \gamma, \quad \ell_{80}^{(1)}=-\frac{i}{2} \mathbf{r}_{+} \cdot \mathbf{r}_{-} \gamma, \quad \ell_{88}^{(0)}=-\frac{N_{c}^{2}}{N_{c}^{2}-1} \mathbf{r}_{+}^{2} \kappa .
\end{gathered}
$$

Wigner transforming the evolution equation of the state evolved by $\lambda_{0}^{\prime}$ gives the Fokker Planck equation

$$
\left(\frac{\partial}{\partial t}+\mathbf{v} \cdot \nabla_{+}\right) \tilde{\rho}_{0}(t)=\left[\frac{\kappa}{4} \nabla_{\mathbf{p}}^{2}+\frac{M}{2} \eta \nabla_{\mathbf{p}} \cdot \mathbf{v}+\frac{\gamma}{2} \mathbf{r}_{+} \cdot \nabla_{\mathbf{p}}+\left(\frac{\gamma}{\sqrt{\kappa}} \frac{\mathbf{r}_{+} \cdot \nabla_{\mathbf{p}}}{2 N_{c}\left|\mathbf{r}_{+}\right|}\right)^{2}\right] \tilde{\rho}_{0}(t),
$$

with corresponding Langevin equation

$$
\frac{\mathrm{d} r_{i}^{+}}{\mathrm{d} t}=\frac{2 p_{i}}{M}, \quad \frac{M}{2} \frac{\mathrm{d}^{2} r_{i}^{+}}{\mathrm{d} t^{2}}=-F_{i}\left(\mathbf{r}^{+}\right)-\eta_{i j} p_{j}+\xi_{i}\left(t, \mathbf{r}^{+}\right)+\theta_{i}\left(t, \mathbf{r}^{+}\right)
$$

We observe the above equation to be similar to the general Langevin equation introduced in Eq. (5) with a number of additional terms. $\kappa$ is here the heavy quarkonium momentum diffusion coefficient defined as the correlator of the momentum kicks $\xi_{i}\left(t, \mathbf{r}_{+}\right)$

$$
\left\langle\xi_{i}\left(t, \mathbf{r}^{+}\right) \xi_{j}\left(t^{\prime}, \mathbf{r}^{+}\right)\right\rangle=\delta\left(t-t^{\prime}\right) \delta_{i j} \kappa .
$$


It is also the heavy quark momentum diffusion coefficient in the form first derived in Ref. [10]. The Einstein relation gives the momentum diffusion coefficient $\eta$ in terms of $\kappa$

$$
\eta_{i j}\left(\mathbf{r}^{+}\right)=\frac{\kappa}{2 M T} \delta_{i j}
$$

$\theta_{i}\left(t, \mathbf{r}_{+}\right)$represents a second random force due to fluctuations in the force between the quark and the antiquark which are on average zero. A similar term occurs in the NRQCD derivation of Blaizot and Escobedo. Its correlator is related to the transport coefficient $\gamma$

$$
\left\langle\theta_{i}\left(t, \mathbf{r}^{+}\right) \theta_{j}\left(t^{\prime}, \mathbf{r}^{+}\right)\right\rangle=\delta\left(t-t^{\prime}\right) \frac{r_{i}^{+} r_{j}^{+} \gamma^{2}}{4 N_{c}^{2} \kappa r_{+}^{2}} .
$$

$F_{i}\left(\mathbf{r}_{+}\right)$is a force related to the correction to the quark-antiquark potential due to $\gamma$

$$
F_{i}\left(\mathbf{r}^{+}\right)=-\gamma \frac{r_{i}^{+}}{2}
$$

No analogous term occurs in Blaizot's and Escobedo's NRQCD calculation.

\section{Conclusion}

In this conference proceeding, we present the derivation of a Langevin equation describing the in-medium evolution of heavy quarkonium. Our method makes use of effective field theory methods and the formalism of open quantum systems to describe the in-medium evolution of heavy quarkonium. We expand to linear order in $E / T$ the master equation derived in [12] which at order 0 in $E / T$ takes the form of a Lindblad equation which was solved in Refs. [12-15]. We follow the procedure of Blaizot and Escobedo of Ref. [16] to obtain a Langevin equation. Our main result, Eq. (34), contains the heavy quark momentum diffusion coefficient $\kappa$ in the form derived by Casalderrey-Solana and Teaney in Ref. [10] as it occurs in the Langevin equation describing a single heavy quark. The Langevin equation, furthermore, contains a term, also occurring in Blaizot's and Escobedo's NRQCD calculation, representing a fluctuating force due to the quarkantiquark potential. Our Langevin equation contains an additional term not present in the Blaizot and Escobedo calculation proportional to the transport coefficient $\gamma$ representing a correction to the quark-antiquark potential. In future works, we plan to show how $\kappa$ as defined in Eq. (7) arises from a similar first principles calculation for a single in-medium quark [17, 18].

\section{References}

[1] Particle Data Group collaboration, P. A. Zyla et al., Review of Particle Physics, PTEP 2020 (2020) $083 \mathrm{C} 01$.

[2] Particle Data Group collaboration, M. Tanabashi et al., Review of Particle Physics, Phys. Rev. D 98 (2018) 030001.

[3] P. Petreczky and J. H. Weber, Strong coupling constant from moments of quarkonium correlators revisited, 2012.06193. 
[4] W. E. Caswell and G. P. Lepage, Effective Lagrangians for Bound State Problems in QED, QCD, and Other Field Theories, Phys. Lett. B 167 (1986) 437.

[5] G. T. Bodwin, E. Braaten and G. P. Lepage, Rigorous QCD analysis of inclusive annihilation and production of heavy quarkonium, Phys. Rev. D 51 (1995) 1125 [hep-ph/9407339].

[6] A. Pineda and J. Soto, Effective field theory for ultrasoft momenta in NRQCD and NRQED, Nucl. Phys. B Proc. Suppl. 64 (1998) 428 [hep-ph/9707481].

[7] N. Brambilla, A. Pineda, J. Soto and A. Vairo, Potential NRQCD: An Effective theory for heavy quarkonium, Nucl. Phys. B 566 (2000) 275 [hep-ph/9907240].

[8] N. Brambilla, A. Pineda, J. Soto and A. Vairo, Effective Field Theories for Heavy Quarkonium, Rev. Mod. Phys. 77 (2005) 1423 [hep-ph/0410047].

[9] H. Breuer and F. Petruccione, The theory of open quantum systems. Oxford University Press, Oxford, 2002.

[10] J. Casalderrey-Solana and D. Teaney, Heavy quark diffusion in strongly coupled $N=4$ Yang-Mills, Phys. Rev. D 74 (2006) 085012 [hep-ph/0605199].

[11] N. Brambilla, M. A. Escobedo, J. Soto and A. Vairo, Quarkonium suppression in heavy-ion collisions: an open quantum system approach, Phys. Rev. D 96 (2017) 034021 [1612.07248].

[12] N. Brambilla, M. A. Escobedo, J. Soto and A. Vairo, Heavy quarkonium suppression in a fireball, Phys. Rev. D 97 (2018) 074009 [1711. 04515].

[13] N. Brambilla, M. A. Escobedo, M. Strickland, A. Vairo, P. Vander Griend and J. H. Weber, Bottomonium suppression in an open quantum system using the quantum trajectories method, JHEP 05 (2021) 136 [2012 . 01240].

[14] H. B. Omar, M. A. Escobedo, A. Islam, M. Strickland, S. Thapa, P. V. Griend et al., QTRAJ 1.0: A Lindblad equation solver for heavy-quarkonium dynamics, 2107.06147.

[15] N. Brambilla, M. A. Escobedo, M. Strickland, A. Vairo, P. V. Griend and J. H. Weber, Bottomonium production in heavy-ion collisions using quantum trajectories: Differential observables and momentum anisotropy, 2107.06222.

[16] J.-P. Blaizot and M. A. Escobedo, Quantum and classical dynamics of heavy quarks in a quark-gluon plasma, JHEP 06 (2018) 034 [1711. 10812].

[17] N. Brambilla, A. Vairo, and P. Vander Griend, Langevin dynamics of in medium heavy quarks and quarkonia, TUM-EFT 117/18.

[18] P. Vander Griend, In medium Langevin dynamics of heavy particles, TUM-EFT 149/21. 